\title{
Generation of Plasma Induced Flow Actuation by DC Glow-like Discharge in a Supersonic Flow
}

\author{
J. Shin*, V. Narayanaswamy*, L. L. Raja ${ }^{\dagger}$, and N. Clemens ${ }^{\ddagger}$ \\ Department of Aerospace and Engineering Mechanics \\ The University of Texas at Austin \\ Austin, TX, 78712-1085, USA
}

\begin{abstract}
Plasma induced flow field modification is studied experimentally using non-equilibrium DC surface discharge in a Mach 3 supersonic flow. An array of small pin electrodes $(\phi=$ $3 / 32$ ") is used in streamwise plasma discharge configuration. The schlieren imaging shows that an oblique shock can be obtained with a volumetric glow discharge on the cathode with a relatively low current (as low as $50 \mathrm{~mA}$ ). The actuation is found to be immediate with a time scale less than $222 \mu$ s whenever there is a noticeable volumetric glow region on the cathode in both cathode upstream and cathode downstream cases. With a laser schlieren, three identifiable modes are observed and the selected modes which have a volumetric glow on top of the cathode can give an actuation while the other mode does not show any noticeable effect. The conditions to get an appropriate mode are dependent mostly on the stagnation pressure. Increasing the current gives a preference to have a constricted plasma while a pressure decrease gives a volumetric glow.
\end{abstract}

\section{Nomenclature}

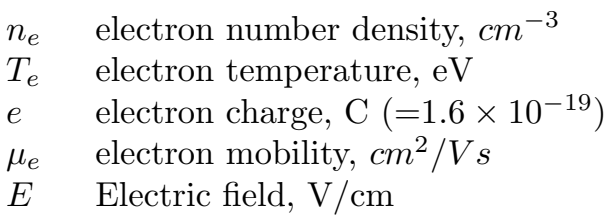

\section{Introduction}

There is growing interest in the use of plasma discharges for control of aerodynamic flows in a variety of speed regimes. A particular class of such discharges called "surface plasma actuators" is used to modify the flow field in the immediate vicinity of a wall boundary. The principal advantage offered by these actuators is the ability to switch them on and off at very high rates. The actuation time scales for the devices can be of the order of microseconds and are essentially instantaneous as far flow dynamics are concerned. A number of recent studies have demonstrated plasma actuation of flows at low speeds as well as at high speeds, using different surface plasma configurations such as dielectric-barrier discharge (DBD) ${ }^{1,2}$ direct-current (DC) glow discharges, ${ }^{3,4}$ radio-frequency (RF) driven glow discharges, ${ }^{5}$ and filamentary arc discharges. ${ }^{6}$

Plasma actuation is physically achieved through one or more of three basic mechanisms: rapid gas heating of the flow due to the plasma (dilitation effect), electrostatic forcing owing to charged species responding to the imposed electric fields in the discharge, and magnetic forcing whereby the plasma experiences a body force owing to electrical currents interacting with an externally imposed magnetic field. For example, the DBD configuration has been shown to effectively use electrostatic forcing to achieve flow actuation, ${ }^{1,2}$ while

\footnotetext{
*Graduate Research Assistant, Student member, AIAA

$\dagger$ Associate Professor, member, AIAA.

$\ddagger$ Professor, Senior member, AIAA.
} 
DC glow and arc discharge use the dilitation effect of gas heating to achieve actuation. ${ }^{3,4}$ MHD has proven effective for controlling high-speed non-equilibrium flows, but very strong magnetic fields are required. ${ }^{7}$ When weaker magnetic fields are present, it appears that the MHD effect is insufficient to show a noticeable change in the flow field. ${ }^{3}$ Scaling studies suggest that at high speeds electrostatic forcing produced by DBD is not as effective as gas heating and therefore recent studies on high speed plasma actuation have focused on the use of intense DC arc discharges. For example, Leonov et al. ${ }^{4}$ reported that significant changes to a supersonic flow field could be made with hot filamentary plasma using a quasi-DC array discharge. A drawback to this type of discharge, however, is that the input power is as high as several kilowatts. Similar study is also made at Mach 5 flow with DC plasma discharge with lower power. ${ }^{3}$

Here, we report a preliminary study on plasma flow actuation in a Mach 3 flow to understand the effect of plasma on the flow field structure. This study is similar to the work by Kimmel et al., ${ }^{8}$ except we are interested in applying the plasma actuators in a different Mach number and pressure regime. In the current case, DC surface discharges are used where the electrodes are arranged in a streamwise-array configuration. Both cathode upstream and cathode downstream cases are considered. The effects of the discharge on the flow are visualized by using schlieren flow visualization.

\section{Experimental Setup}

The experimental work was conducted in a Mach 3 wind tunnel located at The University of Texas at Austin. The windtunnel was built to study supersonic wakes and some modifications were made to it for the current study. The Mach 3 tunnel has a $5 \mathrm{~cm} \times 5 \mathrm{~cm}$ test section and a splitter plate that runs from the plenum section into the test section as shown in Fig. 1. The acrylic splitter plate was $6.3 \mathrm{~mm}$ thick and the plasma actuators were installed on its top surface. The tunnel is equipped with adjustable upper and lower test section walls, which enable the mean pressure gradient to be set. The tunnel is fed by a 500 cu. ft. high-pressure tank and the air is discharged into a $1000 \mathrm{cu}$. ft. vacuum tank. The vacuum tank is evacuated to hold a pressure of 5 - 10 Torr using a Stokes micro vacuum pump (Model: 412H-11) and Roots Connersville rotatory positive vacuum pump (RGS-HVB). The results presented in this paper are

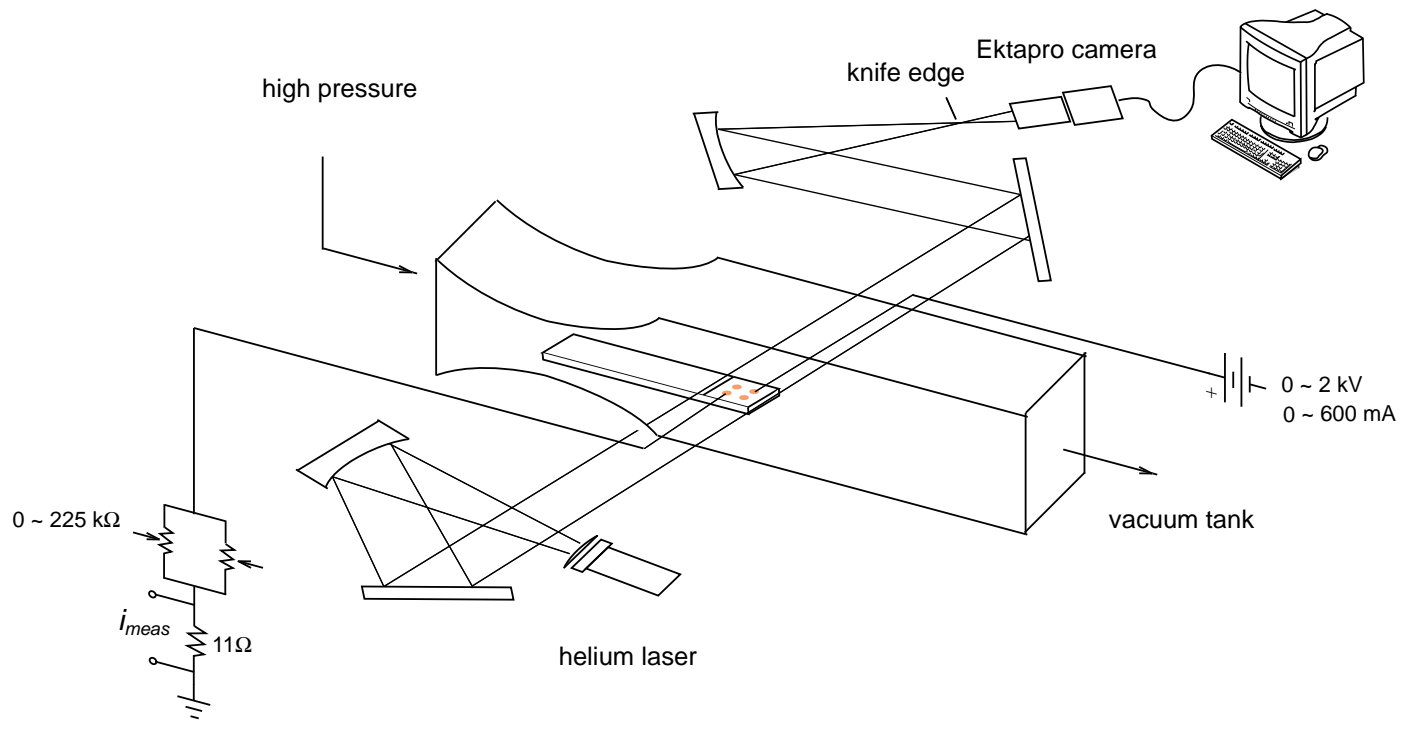

Figure 1. Schematic of experimental setup for a plasma actuation study at a supersonic flow

done at static test section pressures in the range of 16 to 25 Torr. For these conditions, the steady-state operation time was approximately one minute. The static pressure at the test section and the stagnation pressure at the plenum are monitored using PX 241 NG and PX 176 AN (Omega Engineering Inc.) pressure transducers, respectively.

DC surface plasma is generated between two electrodes which are flush-mounted on a ceramic plate. The ceramic plate was inserted into the trailing edge of the splitter plate in the test section. We employ an array of steel pin electrode of diameter of $3 / 32$ " as shown in Fig. 2. Previous work by Menart et al. ${ }^{3}$ indicates 


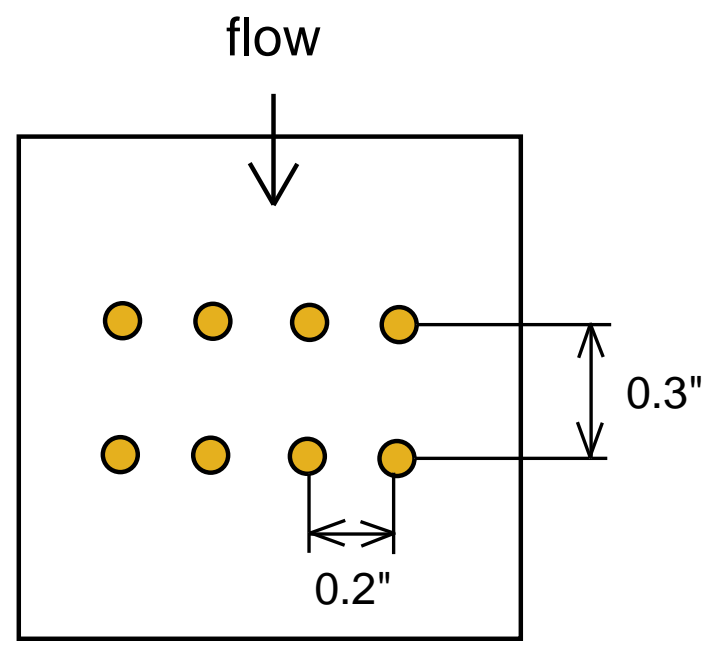

Figure 2. Electrode arrangement for supersonic plasma actuator with an array of steel pin electrodes of diameter 3/32".

that the actuation strength is stronger when the electrodes are arranged in the streamwise direction with the cathode upstream. To check this result both cathode downstream and cathode upstream cases are studied in this work. The discharge is generated by $1.2 \mathrm{~kW}$ high voltage, high current DC power supply (Spellman, SL2PN1200), which can produce a maximum current of $600 \mathrm{~mA}$ and a maximum voltage of $2 \mathrm{kV}$. The plasma current is measured across a $11 \Omega$ resistor and is acquired by data acquisition card (DAQcard ${ }^{\mathrm{TM}}$ AI-16E-4). During laser schlieren imaging the measurement of plasma current is started using a BNC delay generator which is synchronized with the high speed camera to compare the plasma characteristic of each frame with the plasma current data. The sampling frequency for the current measurements was $9 \mathrm{kHz}$ and 18000 samples were taken.

Flashlamp schlieren imaging and laser schlieren imaging were used to visualize the flow field structure in the supersonic flow. The flashlamp was pulsed at $60 \mathrm{~Hz}$ using a BNC delay generator, and the pulse duration of about $2 \mu$ s was small enough to freeze the motion of the flow. The flow was imaged through acrylic side windows on each side of the test section. The light was collimated and focussed by using concave mirrors whose focal length was $3 \mathrm{ft}$. A horizontal razor blade was used as the knife edge. The schlieren images were captured using a Pulnix TM 6710 camera with a framing rate of $60 \mathrm{~Hz}$, triggered internally, and an exposure time of $16 \mathrm{~ms}$. The resolution of the image was $512 \times 480$ pixels. The images were acquired for 30 seconds during which time it was made sure that the tunnel was started. The optical setup for laser schlieren imaging was similar to that for flashlamp schlieren, except a helium-neon laser (5 mW) was used as the light sourece and a high-framing rate CCD camera (Kodak Ektapro 4540mx) was used as the camera. The resolution of the camera was $256 \times 256$ pixels and the framing rate was $4.5 \mathrm{kHz}$ (exposure time of 222 $\mu \mathrm{s})$. The start of the image acquisition was controlled using a BNC delay generator.

\section{Result and Discussion}

\section{A. DC surface plasma}

Figure 3 (a) shows discharge images where the surface plasma is generated on the pin electrode without an external flow. The breakdown voltage for this case is about $700 \mathrm{~V}-1 \mathrm{kV}$ at pressure of 50 Torr in the test section and a driving current is $22 \mathrm{~mA}$. The relatively large volume of glow appears mostly near the cathode. The volume of the glow can vary depending on the local conditions. In the presence of a Mach 3 flow, the glow region shifts downstream and becomes more constricted, as is shown in Fig. 3 (b). Similar observations have been previously reported in the literature. ${ }^{3}$

The electron number density $\left(n_{e}\right)$ of the plasma can be estimated by assuming that the current through the discharge is electron mobility limited. Hence the current density can be represented by $J \simeq e n_{e} v_{d}=e n_{e} \mu_{e} E$. The electron mobility for reduced electric field of $45.9 \mathrm{~V} / \mathrm{cm}$-Torr at 20 Torr of test section pressure can be 


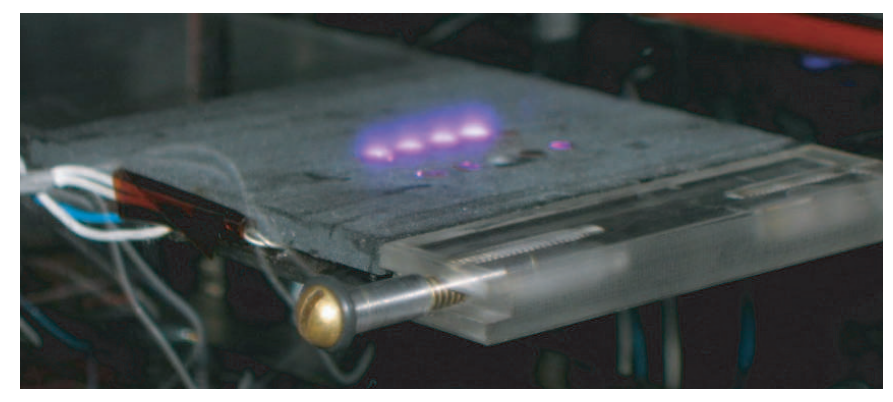

(a)

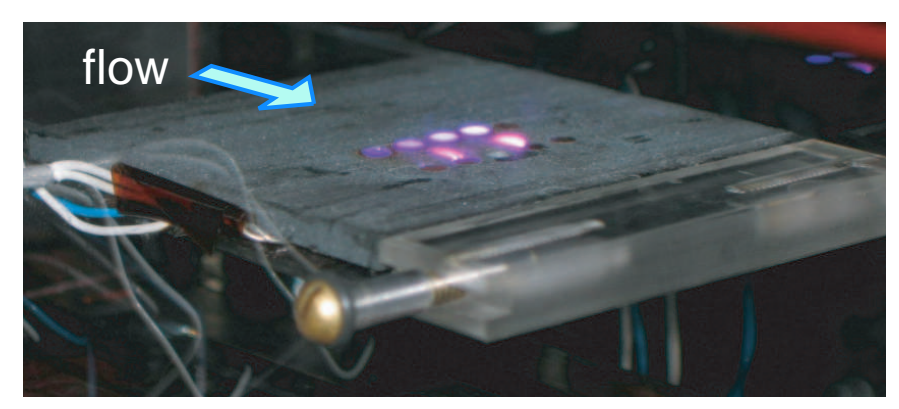

(b)

Figure 3. Plasma discharge images in a supersonic flow. (a) shows the case without a flow and (b) shows when there's a supersonic flow imposed to the plasma

found as $0.45 \times 10^{6} \mathrm{~cm}^{2} / V s$ in the literature. ${ }^{9}$ A typical current density of the discharge in this work is 2.14 $A / \mathrm{cm}^{2}$, which is in the range of the glow discharge regime. For an arc discharge, $J=10^{2}-10^{4} A / \mathrm{cm}^{2} ; 9$ hence, the estimate of electron number density is $6.47 \times 10^{11} \mathrm{~cm}^{-3}$. Assuming quasi-neutrality, the estimate is comparable to Langmuir-probe measurements obtained previously ${ }^{10}$ where the ion number density close to the surface was approximately $10^{11} \mathrm{~cm}^{-3}$.

Electron temperature $\left(T_{e}\right)$ can be estimated as follows. The momentum transfer collision frequency $\left(\nu_{m}\right)$ in air plasma can be found as $7.8 \times 10^{10} \mathrm{~s}^{-1}$ at 20 Torr and the mean free path $(\lambda)$ as $1.5 \times 10^{-3} \mathrm{~cm} .{ }^{9}$ Since the random velocity of electrons $(\bar{v})$ is equal to $\nu_{m} \lambda, T_{e}$ is evaluated as $\sim 3 \mathrm{eV}$. These estimated values suggest that the discharge in this study is in the glow regime, not in the arc regime. Future work will be directed at inferring the temperature of the plasma by using optical emission spectroscopy.

\section{B. Generation of an Oblique Shock and Plasma Discharge Modes in a Supersonic Flow}

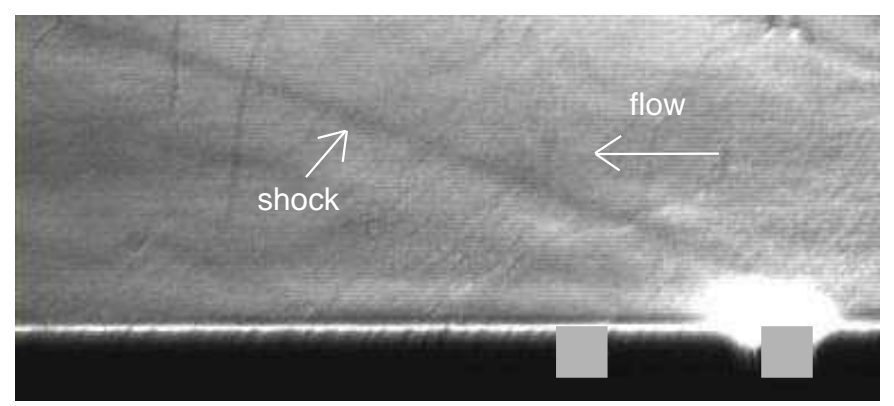

Figure 4. Plasma generated weak oblique shock at Mach 2.76 supersonic flow. Flow is from right to left. Two pairs of electrodes are turned on with cathode upstream and the discharge current of $100 \mathrm{~mA}$ is applied to each pair. The driving voltage is $1 \mathrm{kV}$. Electrode arrangement is shown in a gray block with the cathode to the right. 
Figure 4 shows a flashlamp-schlieren image of the structure of the Mach 3 flow in the presence of a DC surface discharge. A $1 \mathrm{kV}$ driving voltage is applied across the electrodes and each pair delivers $100 \mathrm{~mA}$. The current density is $2.25 \mathrm{~A} / \mathrm{cm}^{2}$. The stagnation pressure is $\sim 500$ Torr. A weak oblique shock is seen to be generated by the glow and the far-field wave angle is about 36 degrees, which corresponds to a Mach number of 2.76. The image shown in Figure 4 is obtained with only two pairs of electrodes being switched on, as this gives a stronger shock, probably because of the higher current density. The oblique shock could also be observed for a discharge using a single electrode pair, although it was not noticeably stronger. The figure clearly shows that the surface plasma can generate a weak shock at relatively low power by forming a localized discharge on the surface. The work of $\mathrm{Kimmel}^{8}$ suggests that the mechanism of shock formation is Joule heating caused by electron-neutral collision. The gas heating expands air in the vicinity of the cathode, which creates a local deflection of the streamlines.
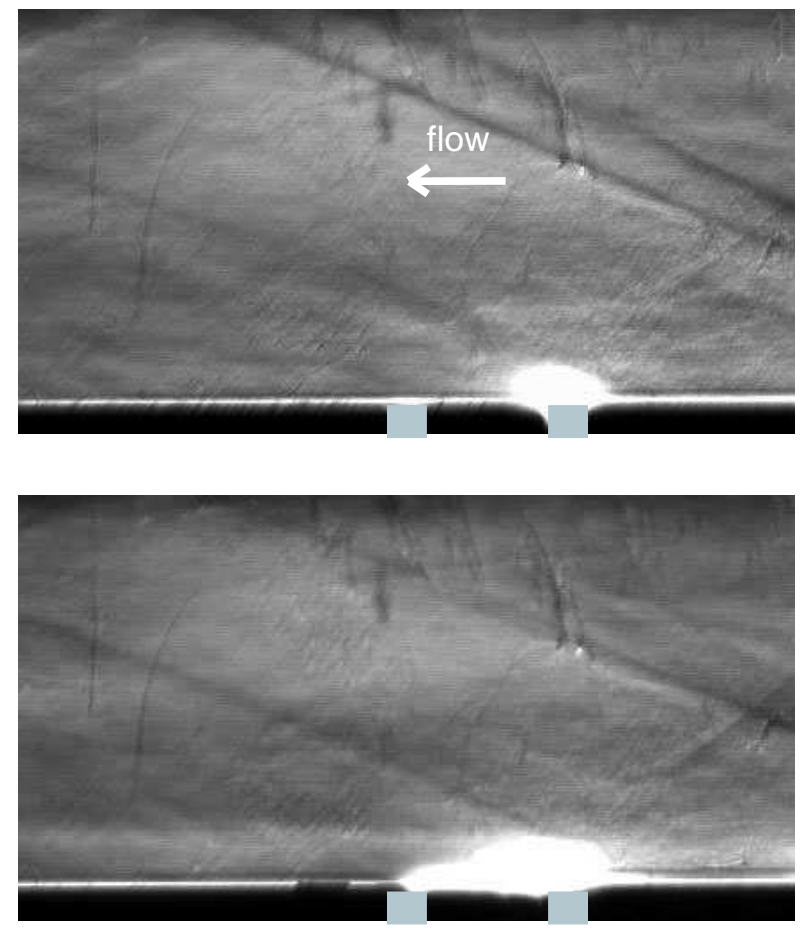

(b)

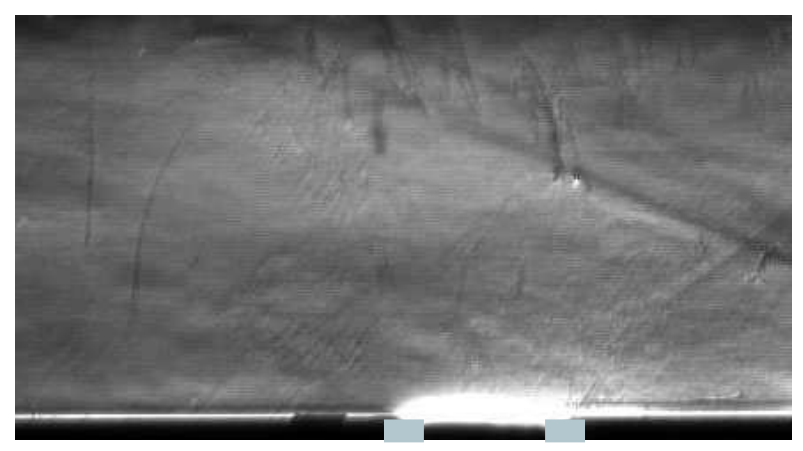

(c)

Figure 5. The weak oblique shock by the different mode of plasma discharge. (a) is for $100 \mathrm{~mA}$ and (b) and (c) are for $150 \mathrm{~mA}$. Flow is from right to left. The weak oblique shocks are visible in (a) and (b) but not in (c).

Figure 5 shows three images that represent three typical modes that were defined based on the shape of the luminous region of the discharge. In Fig. 5 (a) and (b), the oblique shock generated by a plasma is clearly visible with a diffuse, volumetric glow discharge on top of the cathode. In Fig. 5 (c), the plasma is more constricted (streak-like) and no shock is present. The shock intensity is slightly weaker in (b) than in (a) since part of the plasma region is distributed over the electrode, which may make the gas heating less concentrated above the cathode. Similar observations have been reported in the literature which shows that 


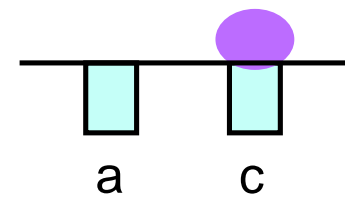

(a)

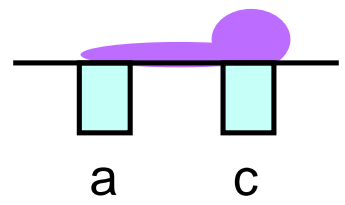

(b)

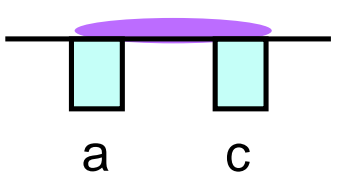

(c)

Figure 6. Different discharge modes in a supersonic flow. 'c' stands for a cathode and 'a' stands for an anode. Flow direction is from right to left. The case (a) has only a volumetric glow on the cathode while case (b) has a volumetric glow on the cathode and a part of the discharge connects to the anode. Case (c) shows a constricted mode where the discharge forms between electrodes.

in the constricted plasma, at even higher current, the pressure increase downstream of the cathode is not as

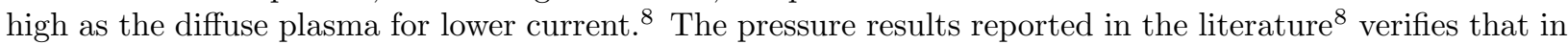
mode (a), the gas heating is more promising and hence the pressure increase is much bigger.

The three modes are shown schematically in Fig. 6. In mode (a), the plasma discharge forms a volumetric glow over the cathode. In mode (b), the discharge is weakly connected to the anode and yet the volumetric glow region appears over the cathode, and in mode (c), the volumetric glow disappears and the discharge remains only between the electrodes. Mode (a) and mode (c) can be distinctly identifiable and the intermediate discharge between mode (a) and mode (c) can be identified as mode (b). The appearance of each mode depends on the several flow parameters and discharge parameters and the modes can be switched from one to the other at the same operation condition. Each discharge mode results in different interaction with a supersonic flow. Mode (a) is also observed in the discharge without a flow, but mode (b) and (c) are observed only in the presence of a flow.

In order to see if the shock is caused by surface heating effects, high speed laser schlieren was used to visualize the flow structure upon an impulsive start of the discharge. These experiments showed that the shock appears within one frame, indicating it is formed within $222 \mu$ sec. Conversely, the oblique shock disappears in the absence of volumetric glow discharge just as rapidly (i.e., the structure changes from (a) or (b) to (c)). This observation shows that the concentrated Joule heating of the supersonic flow occurs rapidly and the effect is not due to surface heating.

By laser schlieren imaging, the discharge is found to be unstable and intermittent as it rapidly switches from one mode to another. Even in steady mode (a), the plasma intensity is found to be growing at a time scale of several milliseconds. The reason for the above observation is probably that as the discharge continues, the gas temperature increases so that the dilitation increases.

The observations in this section are based on images from cathode upstream cases. However, the above observations are found all through even when the electrode polarity is reversed.

\section{Effect of Operating Conditions on the discharge Mode}

In the previous section, we showed that a volumetric glow mode restricted to the vicinity of the cathode produced an oblique shock in the flow field. In this section, we will discuss the effect of different operating conditions on different modes.

As the current flow through the discharge increases, the discharge becomes more intense simultaneously, with an increasing propensity to become constricted (mode(c)). In this constricted mode, the effect of the plasma on the flow is not appreciable and does not show any noticeable change to the flow field. There is an upper limit to the discharge current for an effect to the plasma actuation. However it must be noted that Leonov et $a l .{ }^{4}$ observed significant plasma actuation at much high current with filamentary arc discharge. For our case, as current decreases, the intensity of the oblique shock decreases indicating intermediate current range where the plasma actuation is possible.

For the same stagnation pressure condition, the range of the current inputs which gives mode (a) or (b) is in general larger in cathode downstream case. Mode (b) is however dominant in cathode downstream case. It is found that the occurrence of volumetric glow is found to be strong at near $200 \mathrm{~mA}$ in cathode upstream and $300 \mathrm{~mA}$ in cathode downstream. For fixed input power, the cathode upstream case is more effective than cathode downstream case to the flow structure in producing the perturbation. 
As discussed in the previous section, the mode (a) tends to occur at lower stagnation pressure. The minimum stagnation pressure for our wind tunnel is about 450 Torr to sustain a supersonic flow which corresponds to a static pressure of 16 Torr. At this pressure, the plasma structure exhibits mode (a) over much of the plasma current range tested. As the stagnation pressure increases up to 690 Torr (25 Torr static pressure), the occurrence of mode (a) becomes infrequent and the size of the volumetric glow decreases. The mode shifts towards mode (c) with increasing stagnation pressure. Consequently the presence of oblique shock is not likely at higher stagnation pressures. A change in the stagnation pressure changes the size of a volumetric glow on cathode and increases the discharge instability. With a higher momentum flow at higher stagnation pressure, the discharge mode becomes more constricted. In cathode downstream case, the effect of stagnation pressure is weak. The plasma discharge structure in all cathode downstream cases is more likely in mode (b) rather than mode (a). However, even in cathode downstream case, as long as there's a volumetric glow on the cathode, the weak oblique shock can be obtained, while the shock intensity is not as high as cathode upstream case otherwise at the similar operating conditions.

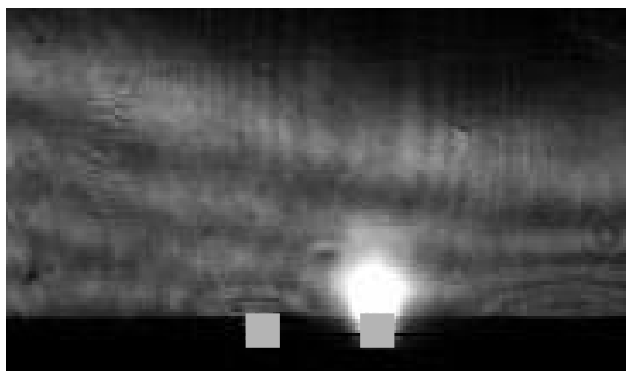

(a)



(b)

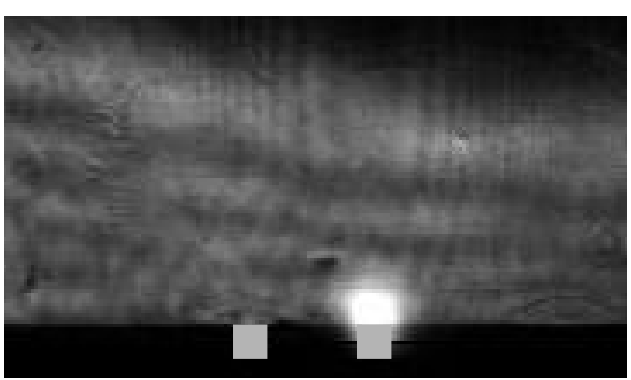

(c)

Figure 7. Laser schlieren image of the plasma discharge without imposing a supersonic flow. Corresponding pressures are 16.8, 20.32, and 25.4 Torr from left to right. Electrode arrangement is shown as a gray block with a cathode on the right of each image.

As the stagnation pressure becomes lower, the plasma becomes more diffuse due to the larger mean free path. Figure 7 shows the image of plasma discharge without a flow. All three images show the diffuse volumetric glow discharge on the cathode with a varying size of the glow. Figure 7 (a) is for a static pressure 
of 16.8 Torr and Fig's 7 (b) and (c) are for 20.32 Torr and 25.4 Torr, respectively. The figure indicates that the size of the volumetric glow region shrinks with an increasing pressure. A change of 10 Torr in pressure reveals a noticeable change in size of the glow. Generally, 10 Torr difference in pressure is not a considerable change. However, at low pressure this change seems appreciable. This pressure effect and the fact that the flow makes the discharge more constricted decreases the tendency to have a mode (a) at higher pressures.

Turbulence is probably another contributing factor. In the presence of a flow, increasing stagnation pressure increases mass flow rate. A higher mass flow rate results in higher Reynolds number and may cause the flow too become turbulent. The effect of turbulence on the plasma is studied by forcing a transition with a rough sandpaper upstream of the plasma actuator. This result is compared with the case without a sandpaper, for otherwise under identical conditions. It appears that the presence of the sandpaper does not change the discharge structure significantly, indicating that the flow in the test section is largely turbulent for all of the cases discussed above.

Finally, the transient current profile is monitored while the schlieren image is taken. Transient current

(a)

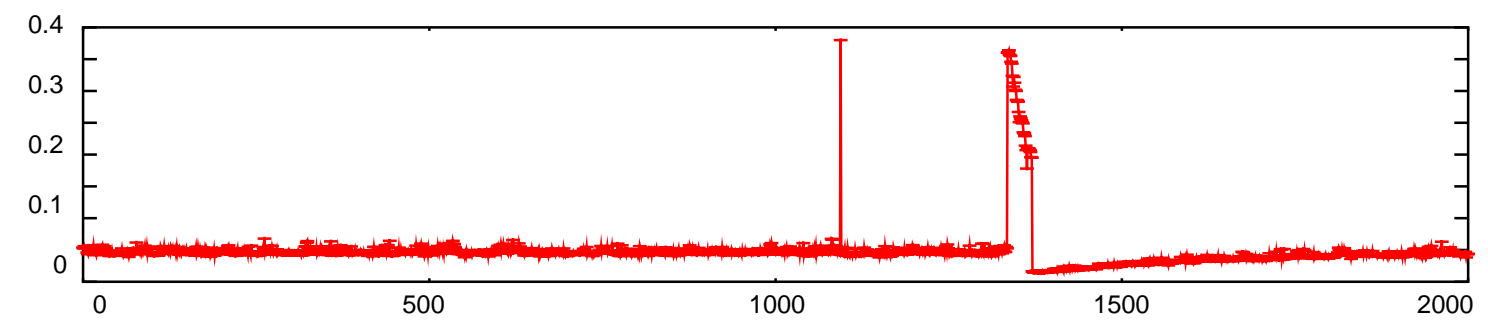

(b)

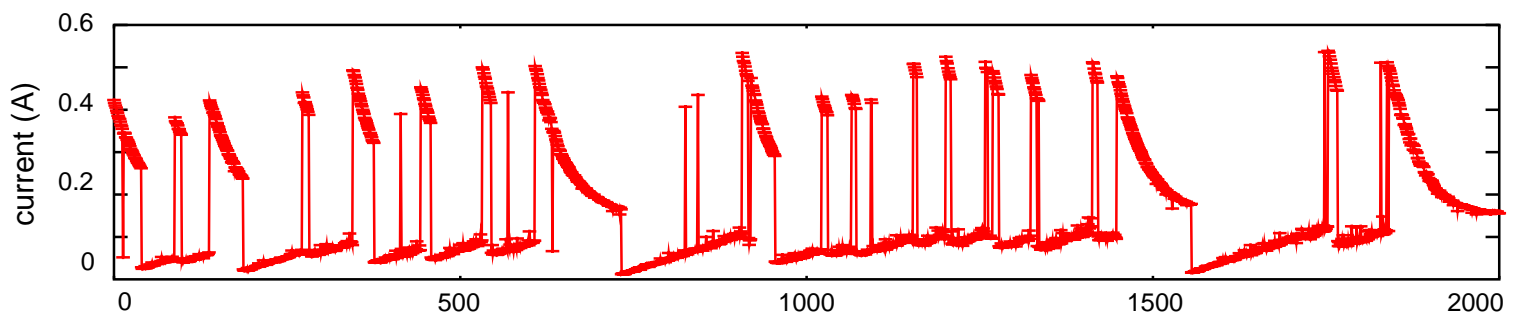

(c)

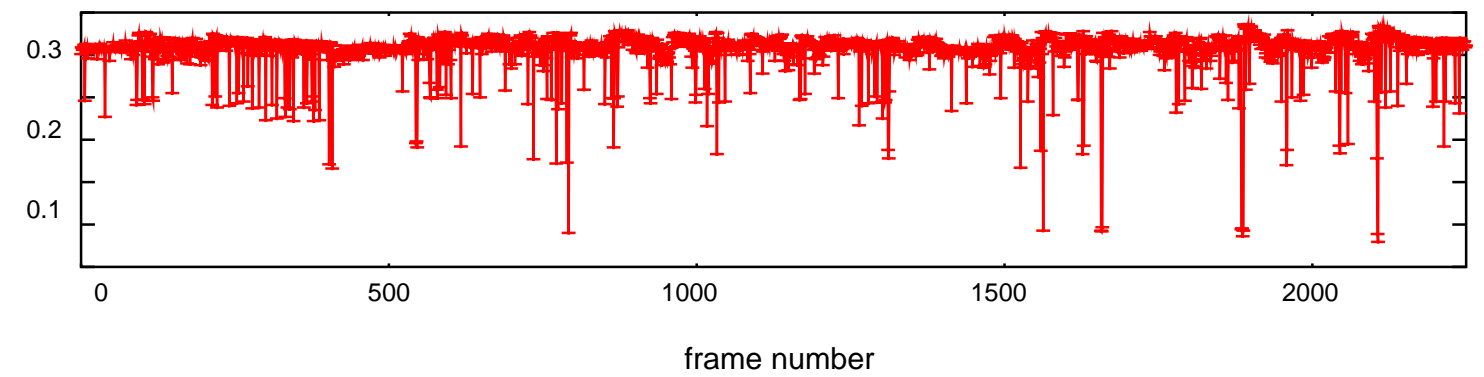

Figure 8. Time evolution of current data in a supersonic flow. (a) is for $50 \mathrm{~mA}$ and (b) is for $150 \mathrm{~mA}$ at a stagnation pressure of 472 Torr. (c) is for $300 \mathrm{~mA}$ at a stagnation pressure of 600 Torr. All three cases are for 0.25 seconds of duration.

is shown in Figure 8. Figure 8 (a) is for a $50 \mathrm{~mA}$ set-point current of power supply and 8 (b) is for $150 \mathrm{~mA}$ set-point current of power supply at a stagnation pressure of 472 Torr. Figure 8 (c) is for $300 \mathrm{~mA}$ set-point current of power supply at a stagnation pressure of 600 Torr. All three transients are for a duration of 0.25 seconds. In the $50 \mathrm{~mA}$ set-point case, the discharge current data shows the most stable plasma with the discharge throughout in mode (a). The sudden spikes of the current are probably caused by plasma instability. In the $150 \mathrm{~mA}$ set-point case, the discharge current starts to fluctuate and this fluctuation is reflected in the discharge mode. In this case mode (a) switches to mode (b) or (c). There are two distinct regime in the current waveform. First, the current grows gradually and before it reaches its set-point value it increases sharply. This is observed in the image as the gradual increase of the size of the volumetric glow discharge. Once current increases sharply, it gradually decreases and drops to lower current and repeats 
the same process again. During the stage when the current increases, the plasma is in mode (a), but in a decaying regime, it is likely to be in mode (b) or mode (c). In $300 \mathrm{~mA}$ set-point case, the current transience is more stable than $150 \mathrm{~mA}$ set-point case in that the discharge current remains at $300 \mathrm{~mA}$ with sudden decrease in the current. In this case the discharge remains in mode (c) through most of the transience.

\section{Conclusions}

An experimental study of the plasma-induced flow field modification was conducted in the Mach 3 wind tunnel facility of the Center for Aero-mechanics Research at University of Texas, Austin. DC surface plasma with small pin electrodes was used. Schlieren imaging was used to visualize the flow field structure. It was observed that under certain pressure and plasma current conditions, a weak oblique shock was formed immediately after switching on the plasma. High speed laser schlieren, with a $4.5 \mathrm{kHz}$ frame rate, showed that the response time is less than $222 \mu \mathrm{sec}$. It was also found that the structure of plasma discharge can be typically classified in three different modes:

- mode (a) in which the concentrated volumetric glow discharge region sits on top of the cathode

- mode (b) in which part of the the discharge is concentrated on top of the cathode and other constricted between cathode and anode

- mode (c) in which the entire plasma discharge is constricted between cathode and anode

Of the above mentioned modes, mode(a) and (b) were found to give an immediate actuation while mode (c) did not show any visible changes to the flow field. It was proposed that this significant actuation is due to concentrated Joule heating of the gas on top of the cathode and the Joule heating is directly related to volumetric discharge found in mode (a) and (b).

Experiments showed that it is possible to sustain the volumetric discharge and hence the weak oblique shock at lower values of stagnation pressure and plasma current. The effect of flow on plasma was primarily in constricting the plasma between cathode and anode. Increase in plasma current made the plasma more constricted. At high currents, the plasma was mostly in the mode (c) and was stable. Increase in pressure uniformly compressed the volumetric glow and increased the tendency to switch the dominant mode from mode (a) or (b) to mode (c). For a given current the plasma discharge changes its mode for a relatively small change (several Torr's) in static pressure. Basically, the plasma becomes more diffusive at lower pressure.

\section{Acknowledgments}

This study was supported by an AFOSR-MURI grant (FA 9550-04-1-0387).

\section{References}

\footnotetext{
${ }^{1}$ Roth J. R., "Aerodynamic flow acceleration using paraelectric and peristaltic electrohydrodynamic effects of a One Atmosphere Uniform Glow Discharge Plasma," Phys. of Plasmas, Vol. 10, No. 5, May 2003.

${ }^{2}$ Enloe C. L., McLaughlin T. E., VanDyken R. D., Kachner K. D., Jumper E. J., Corke T. C., Post M., and Haddad O., "Mechanisms and Responses of a Single Dielectric Barrier Plasma Actuator: Geometric Effects," AIAA Journal, Vol. 42, No. 3, March 2004.

${ }^{3}$ Menart J., Shang J., Kimmel R. and Hayes J., "Effects of Magnetic Fields on Plasmas Generated in a Mach 5 Wind Tunnel," 34th AIAA Plasmadynamics and Lasers Conference, AIAA-2003-4165, June 2003.

${ }^{4}$ Leonov S., Bityurin V., Savelkin K. and Yarantsev D., "Effect of Electrical Discharge on Separation Processes and Shocks Position in Supersonic Airflow," 40nd AIAA Aerospace Sciences Meeting and Exhibit, AIAA-2002-0355, January 2002.

${ }^{5}$ Merriman, S., Ploenjes, E., Palm, P., and Admovich, I., "Shock Wave Control by Nonequilibrium Plasmas in Cold Supersonic Gas Flows," AIAA Journal, Vol. 39, No. 8, August 2001.

${ }^{6}$ Samimy, M., Adamovich, I., Webb, B., Kastner, J., Hileman, J., Keshav, S., and Palm, P., ”Developement and Application of Localized Arc Filament Plasma Actuators for Ject Flow and Noise Control," 42nd AIAA Aerospace Sciences Meeting and Exhibit, AIAA-2004-184, January 2002.

${ }^{7}$ Macheret, S. O., Shneider, M. N., and Miles, R. B., "Magnetohydrodynamic and Electrohydrodynamic Control of Hypersonic Flows of Weakly Ionized Plasmas," AIAA Journal, Vol. 42, No. 7, July 2004.

${ }^{8}$ Kimmel, R., Hayes, J., Menart, J., and Shang, J., "Effect of Surface Plasma Discharges on Boundary Layers at Mach 5," 42nd AIAA Aerospace Sciences Meeting and Exhibit, AIAA-2004-509, January 2002.

${ }^{9}$ Raizer, Yu.P., Gas Discharge Physics, Springer, New York, 1991.
} 
${ }^{10}$ Menart, J., Henderson, S., Atzbach, C., Shang, J., Kimmel, R., and Hayes, J., "Study of Surface and Volumetric Heating Effects in a Mach 5 Flow," 35th AIAA Plasmadynamics and Lasers Conference, AIAA-2004-2262, 28 June - 1 July 2004.

10 of 10 